\title{
Facile Synthesis of 9-Substituted 9-Deazapurines as Potential Purine Nucleoside Phosphorylase Inhibitors
}

\author{
Hsiencheng SHIH, ${ }^{*}$ Howard Brinkerhoff CotTAm, and Dennis Anthony CARSON \\ Department of Medicine and The Sam and Rose Stein Institute for Research on Aging, University of California, San Diego, \\ La Jolla, California 92093-0663, U.S.A. Received October 19, 2001; accepted January 4, 2002
}

\begin{abstract}
A facile synthesis of 9-substituted 9-deazapurines as potential inhibitors of purine nucleoside phosphorylase has been achieved by the direct Friedel-Crafts aroylation or arylmethylation of 9-deazapurines using trifluoromethanesulfonic acid as catalyst. The aroylated 9-deazapurines could be transformed into the corresponding 9-arylmethyl derivatives by the Wolff-Kishner reaction. A novel synthesis of 9-deazahypoxanthine was also developed by treatment of 4-hydroxy-5-phenylazo-6-methylpyrimidin-2-thione with triethyl orthoformate in trifluoroacetic acid (TFA) to yield 8-oxo-7H-2-phenylpyrimido[5,4-c]pyridazin-6-thione followed by Raney nickel reduction.
\end{abstract}

Key words 9-deazahypoxanthine; 9-deazapurine; 9-aroyl-9-deazapurine; 9-arylmethyl-9-deazpurine; purine nucleoside phosphorylase inhibitor

Purine nucleoside phosphorylase (PNP) catalyzes reversibly the phosphorolysis of guanosine, inosine, and their $2^{\prime}$-deoxyribonucleoside derivatives to the corresponding free base, $\alpha$-ribose-1-phosphate, or 2-deoxy- $\alpha$-ribose-1-phosphate. Inhibition of PNP may cause the selective accumulation of dGTP, ${ }^{1)}$ selective depletion of T lymphocytes, and inhibition of cellular immune response. ${ }^{2)}$ Thus, a potent PNP inhibitor may provide a new and effective immunosuppressive agent in the treatment of T-cell dependent diseases such as rheumatoid arthritis, psoriasis, T-cell leukemia and lymphoma. Various derivatives of 9-substituted 9-deazaguanine and 9-deazahypoxanthine as potent competitive inhibitors of purine nucleoside phosphorylase based on the application of crystallographic and modeling methods have been described. ${ }^{3-7)}$ All these derivatives were prepared by multi-step synthesis without using a common building block. Usually, the last step in these processes was the construction of the pyrimidine or pyrrole ring of the deazapurine base. ${ }^{3-7)}$ In order to find a simple and effective method to explore more PNP inhibitors, a facile synthesis of these analogues was developed based on the strategy of the direct aroylation or arylmethylation of available building blocks, such as 9-deazaxanthine $\left.(\mathbf{1}),{ }^{8}\right)$ 9-deazaguanine $(\mathbf{2}),{ }^{8,9)}$ or 9-deazahypoxanthine (3). ${ }^{9)}$ The aroylated compounds could be converted to the corresponding arylmethyl derivatives or vise versa. These approaches would be much more efficient than the previously reported multi-step synthesis which employed no common building blocks. Herein, we report a facile synthesis of 9substituted 9-deazapurines and an improved method to prepare 9-deazahypoxanthine.

\section{Results and Discussion}

Synthesis of 9-Substituted 9-Deazaxanthine As C-deoxyribonucleosides ${ }^{10)}$ had been prepared by the direct C-glycosylation of 7- and 9-deazaguanine using stannic chloride as catalyst, it was possible to synthesize 9-substituted 9deazapurines by a direct aroylation or arylmethylation of available 9-deazaxanthine (1), 9-deazaguanine (2) or 9-deazahypoxanthine (3). In our initial attempts, the Friedel-Crafts benzoylation of 1 in the presence of Lewis acid catalysts gave a mixture of 9-deazaxanthine benzoylated at $\mathrm{N}-1, \mathrm{~N}-3$, and N-7 as major products. As acylation of olefins could pro- ceed through carbocation intermediate in the presence of strong acids, ${ }^{11)}$ treatment of $\mathbf{1}$ with benzoyl chloride in polyphosphoric acid at $100{ }^{\circ} \mathrm{C}$ gave unsatisfactory results. However, acylation of 1 with benzoyl chloride using triflic acid as catalyst in nitromethane, provided the desired 9-benzoyl-9-deazaxanthine, 4 , in low yield. Optimization of the reaction conditions for benzoylation was achieved upon the reaction of 1 with benzoyl chloride (1.2 eq), using triflic acid $(>10$ eq) as catalyst and solvent at temperatures higher than $60{ }^{\circ} \mathrm{C}$ for $48 \mathrm{~h}$ providing $75 \%$ yield of 4 . A similar procedure was applied to yield 9-( $p$-methylbenzoyl)- and 9-(3',4'dichlorobenzoyl)-9-deazaxanthine, 5 and $\mathbf{6}$, respectively. In these reactions, one major aroylated product at the C-9 position was isolated. 8-Aroyl or 8,9-diaroyl derivatives were not found. The lack of these side products indicated the exclusive aroylation at C-9. The electron-withdrawing aroyl substituent at C-9 might play an important role to deactivate further aroylation at the C-8 position of 9-deazaxanthine. However, a limitation in the scope of this electrophilic substitution was observed. We found that reactions involving aroyl chlorides bearing electron-withdrawing groups at the para position, proceeded with great difficulty due to the limited availability of in-situ generated carbocation. We then investigated several procedures to convert the aroyl substituent to arylmethyl derivatives. Attempts to reduce $\mathbf{4}$ by the Clemmenson reduction or by catalytic hydrogenation using $10 \%$ palladium on carbon under acidic or basic conditions, failed to give product. However, it was found that $\mathbf{4}$ could be reduced to 9-benzyl-9-deazaxanthine, 7 (56\% yield), by the Wolff-Kishner reaction, using 10 eq of $\mathrm{NaOH}$ and 10 eq of hydrazine in ethylene glycol at $100^{\circ} \mathrm{C}$. Similarly, 9-( $p$ methylbenzyl)-9-deazaxanthine, 8 , was obtained from 5 by the Wolff-Kishner reaction. The successful synthesis of 9aroyl-9-deazaxanthine led us to turn our attention to the preparation of 9-arylmethyl derivatives by the direct Friedel-Crafts alkylation of $\mathbf{1}$. Previously, the Friedel-Crafts alkylation of $\mathbf{1}$ with benzyl bromide using a Lewis acid catalyst such as stannic chloride, or aluminum chloride in nitromethane, or acetonitrile, produced a mixture of 9-deazaxanthines benzylated at C-8, C-9, N-1, or N-7. Attempts to prepare the desired compound using silver oxide as catalyst ${ }^{12)}$ which had been successfully used in an indole system, 
resulted in benzylation at N-7 and C-8 positions. Treatment of $\mathbf{1}$ with benzyl alcohol, a good source of benzyl cation, ${ }^{13,14)}$ in triflic acid at room temperature yielded 8,9-dibenzylated derivatives. The reaction of $\mathbf{1}$ with benzyl bromide in triflic acid as catalyst and solvent at $60-100^{\circ} \mathrm{C}$ also gave 8,9dibenzyl derivatives. In an effort to solve the problem, treatment of 1 with benzyl bromide using triflic acid ( $>4$ eq) as catalyst in glacial acetic acid at $100^{\circ} \mathrm{C}$ or TFA at $80^{\circ} \mathrm{C}$ for $48 \mathrm{~h}$, resulted in 7 as a major product. Similar results were obtained by the same approach to yield $\mathbf{8}$ and $\mathbf{9}$, respectively. However, the better result of $\mathbf{9}$ was obtained when aluminum chloride, a Lewis acid, was added as the second catalyst to increase the formation of 3,4-dichlorobenzyl cation. In these reactions, acetic acid or TFA not only helped to dissolve 9deazaxanthine but also increased the formation of benzyl cation for alkylation. We isolated 8,9-diarylmethylated compounds as a minor product. However, we observed no 8-arylmethyl-9-deazaxanthin. It was postulated that arylmethylation occurred first at C-9, followed by further electrophilic substitution at C-8 to yield 8,9-diarylmethyl derivatives.

Synthesis of 9-Substituted 9-Deazaguanine Unlike 1, acylation of 2 can occur at 2 -amino and C-9 positions. Thus, reaction of 2 with 2.2 eq of benzoyl chloride in triflic acid $\left(>20\right.$ eq) at $60^{\circ} \mathrm{C}$ resulted in dibenzoyl derivative at 2-amino and C-9 positions, which could be converted to the desired 9benzoyl-9-deazaguanine, 10, by treatment of the reaction mixture with excess $\mathrm{NaOH}$ (>40 eq) for $2 \mathrm{~h}$ at $60^{\circ} \mathrm{C}$, followed by neutralization. However, at a higher temperatures $\left(>80^{\circ} \mathrm{C}\right)$, 2-acylamide of dibenzoyl derivative was further hydrolyzed by triflic acid to give 10. Similar procedure by treatments of $\mathbf{2}$ with $p$-methylbenzoyl chloride, 3,4dichlorobenzoyl chloride, 3,5-dichlorobenzoyl chloride, or 3nitrobenzoyl chloride resulted in $\mathbf{1 1}, \mathbf{1 2}, \mathbf{1 3}$, or 14, respectively. However, treatment of $\mathbf{2}$ with $p$-methoxybenzoyl chloride at $80^{\circ} \mathrm{C}$ for $40 \mathrm{~h}$ resulted in 9-( $p$-hydroxybenzoyl)-9deazaguanine, 15, because $p$-methoxyl substituent was further hydrolyzed by triflic acid. We found that the reaction gave a major product of 9-( $p$-methoxybenzoyl)-9-deazapurine $\left(\mathrm{ms}, 285, \mathrm{MH}^{+}\right)$at a lower temperature $\left(60^{\circ} \mathrm{C}\right)$ for $20 \mathrm{~h}$, but failed to give a product at higher temperature $\left(>100^{\circ} \mathrm{C}\right)$ for $24 \mathrm{~h}$, due to the early cleavage of the methoxyl group of $p$-methoxybenzoyl chloride by triflic acid before it could be acylated at C-9. The chemistry of electrophilic substitution of 9-deazaguanine under strong acidic condition was similar to that of 9-substituted 9-deazaxanthine. Accordingly, treatment of 2 with 1.2 eq of benzyl, $p$-methylbenzyl, 3,4dichlorobenzyl, 3-nitrobenzyl bromide, or $p$-methoxybenzyl chloride and 5 eq of triflic acid in glacial acetic acid or TFA offered $16,17,18,19$, or 20 as a major product, respectively.

Synthesis of 9-Deazahypoxanthine and Its 9-Substituted Derivatives Previously, 3 had been prepared by dichlorination of $\mathbf{1}$ with phosphorus oxychloride followed by partial alkaline hydrolysis of 6-chloro substituent and the subsequent removal of 2-chloro by hydrogenation with palla- dium on carbon, ${ }^{9)}$ or by the condensation of isoxazole and diethyl aminomalonate to form pyrrole derivative followed by reaction with formamidine acetate, ${ }^{15)}$ or by palladium-catalyzed cross-coupling of 4-methoxy-5-nitro-6-iodopyridine and trimethyl(tributylstannylethynyl)silane to form 4-methoxy5-nitro-6-trimethylsilylethynylpyridine followed by construction of an annulated pyrrole ring. ${ }^{16)}$ However, in the routes to synthesize intermediates of 1 and 2, 6,8-dioxo-7H-2phenylpyrimido[5,4-c]pyridazine ${ }^{8)}$ and 6-amino-8-oxo-2phenylpyrimido[5,4-c]pyridazine, ${ }^{8)}$ respectively, by modifying Klein's procedure ${ }^{8}$ using TFA as solvent, we found a simple two-step synthesis of $\mathbf{3}$ with an overall yield of $50 \%$. This new method involved the reaction of 4-hydroxy-5phenylazo-6-methylpyrimidin-2-thione, $\mathbf{2 5},{ }^{17)}$ with triethyl orthoformate in TFA at $70^{\circ} \mathrm{C}$ to give 8 -oxo- $7 \mathrm{H}$-2phenylpyrimido[5,4-c]pyridazin-6-thione, 26. The subsequently concomitant ring constriction and removal of the 2thione of this intermediate with Raney-nickel in methanol offered 3 (Chart 2). In this reaction, hydrazine or $\mathrm{NaOH}$ could be used as base catalyst. The chemistry of $\mathbf{3}$ toward the Friedel-Crafts acylation or alkylation was expected to be the same as 1. Accordingly, 9-benzoyl-9-deazahypoxanthine (21), 9-(3',4'-dichlorobenzoyl)-9-deazahypoxanthine (22), 9-benzyl-9-deazahypoxanthines (23) and 9-(3',4'-dichlorobenzyl)9-deazahypoxanthine (24) were obtained by the same procedures used in the preparations of 9-substituted 9-deazaxanthines.

Summary We had developed a novel synthesis of 9deazahypoxanthine and demonstrated more efficient and concise approaches to prepare 9-substituted 9-deazapurines via the Friedel-Crafts acylation or alkylation, using triflic acid as catalyst. These approaches were based on the in-situ generation of available carbocation or arylmethyl cation, so that aroylation or arylmethylation could occur at the C-9 of 9deazapurine. The aroylated compounds could be transformed into the corresponding arylmethyl analogues by the Wolff-Kishner reaction or vise verse as evidenced by the

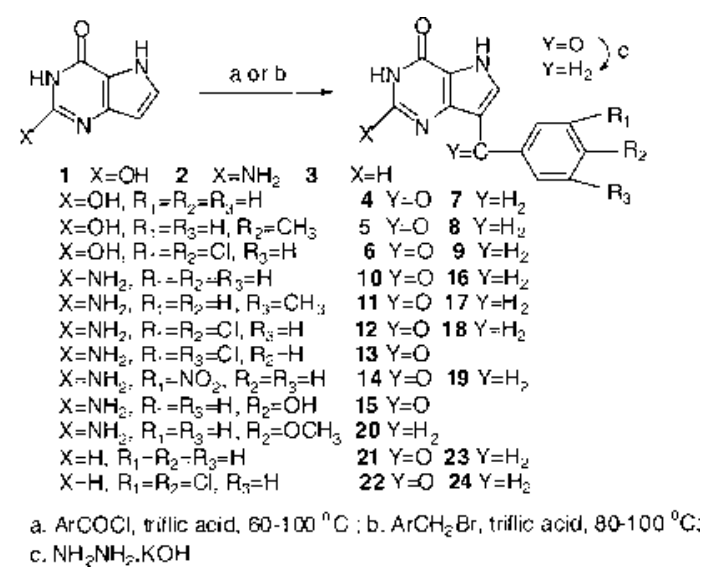

Chart 1

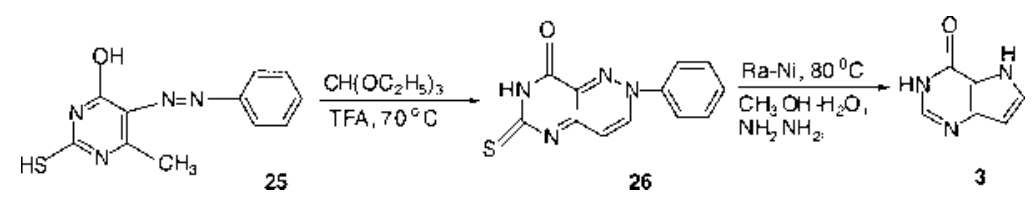


conversion of $\mathbf{1 6}$ to $\mathbf{1 0}$ using $\mathrm{CrO}_{3}$ in acetic acid. ${ }^{18)}$ We are currently applying these methods to synthesize 7-substituted 7-deazapurines.

\section{Experimental}

All solvents and chemicals were of analytical grade. Melting points were determined by the capillary method with a MEL-Temp II apparatus, and uncorrected. NMR spectra in DMSO- $d_{6}$ were recorded at $500 \mathrm{MHz}$. Mass spectrum was obtained by FAB-MS.

General Procedures. Method A. Aroylation of 9-Deazapurines 1: The mixture of $\mathbf{1}(200-300 \mathrm{mg}$ as $1.0 \mathrm{eq})$ or $\mathbf{3}(100 \mathrm{mg}$ as $1.0 \mathrm{eq})$, and aroyl chloride $(1.2 \mathrm{eq})$ in triflic acid $(10 \mathrm{eq})$ was stirred at $60-100^{\circ} \mathrm{C}$ for $48 \mathrm{~h}$. After cooling, water was added to the reaction mixture. The resulting precipitate was filtered and washed with solvent several times sach as methylene chloride, ethyl acetate, acetone, or methanol, to give desired product. Further purification can be done by repeatly boiling the product in methanol and then filtered several times.

2: The mixture of $2(200-300 \mathrm{mg})$ and aroyl chloride (2.2 eq) in triflic acid (>20 eq) was stirred at $80-120^{\circ} \mathrm{C}$ for $48 \mathrm{~h}$. After cooling, water was added to the reaction mixture. The resulting precipitate was filtered and washed several times with solvent sach as methylene chloride, ethyl acetate, acetone, or methanol, to give desired product. Further purification can be done by repeatly boiling the product in methanol and then filtered several times.

B. Arylmethylation of 9-Deazapurines The mixture of 9-deazapurine $(100-300 \mathrm{mg})$, arylmethyl bromide $(1.2 \mathrm{eq})$ and triflic acid (4-10 eq) in acetic acid $(10 \mathrm{ml})$ or TFA $(10 \mathrm{ml})$ was stirred at $80-100^{\circ} \mathrm{C}$ for $48 \mathrm{~h}$. The reaction mixture was concentrated under vacuo. The residue was added with water, neutralized and then extracted with ethyl acetate twice. The combined organic phase was dried and evaporated. The residue was purified by flash column chromatography using 5\% methanol in methylene chloride as an eluent to give product. For the synthesis of $9-\left(3^{\prime}, 4^{\prime}\right.$-dichlorobenzyl $)-9$ deazapurines, a modification was made by adding aluminum chloride (2.2 eq) as the second catalyst to increase the formation of 3,4-dichlorobenzyl cation.

C. The Wolff-Kishner Reaction The mixture of 9-deazapurine (100$150 \mathrm{mg}), \mathrm{NaOH}(10 \mathrm{eq})$ and hydrazine $(10 \mathrm{eq})$ in ethylene glycol $(10 \mathrm{ml})$ was stirred at $100-120^{\circ} \mathrm{C}$ for $16 \mathrm{~h}$. The reaction mixture was diluted with water and then neutralized with acetic acid. The mixture was then extracted with ethyl acetate twice. The combined organic phase was dried and evaporated. The residue was chromatographed using $10 \%$ methanol in methylene chloride as an eluent to give the product.

3H,5H-Pyrrolo[3,2-d]pyrimidin-4-one (3): The mixture of 4-hydroxy-5phenylazo-6-methylpyrimid-2-thione ${ }^{16)}(12 \mathrm{~g})$, triethyl orthoformate $(100 \mathrm{ml})$ in TFA $(60 \mathrm{ml})$ was stirred at $70^{\circ} \mathrm{C}$ overnight. After cooling, the reaction mixture was filtered and washed with $\mathrm{CH}_{2} \mathrm{Cl}_{2}$ to give $7.0 \mathrm{~g}$ of crude 8-oxo7H-2-phenylpyrimido[5,4-c]pyridazin-6-thione [NMR, $\delta 7.70(\mathrm{~m}, 3 \mathrm{H}$, aromatic H), 7.90 (m, 2H, aromatic H), 8.15 (bs, $1 \mathrm{H}, \mathrm{C}-4 \mathrm{H}), 9.2$ (bs, $1 \mathrm{H}, \mathrm{C}-$ $3 \mathrm{H}), 9.57(\mathrm{~d}, 1 \mathrm{H}, \mathrm{NH})]$. This crude intermediate in $120 \mathrm{ml}$ of $50 \%$ methanol-water and $5 \mathrm{ml}$ of hydrazine was added Ra-Ni (wet, $40 \mathrm{~g}$ ) with stirring at $80^{\circ} \mathrm{C}$ overnight. The reaction mixture was filtered and the filtrate was evaporated to give almost pure product $(3.22 \mathrm{~g}, 49 \%)$, which was recrystallized from hot water to give pure compound. $\mathrm{mp}>300^{\circ} \mathrm{C}$ (lit. $.^{9)}>300{ }^{\circ} \mathrm{C}$ ); MS $m / z, 136\left(\mathrm{MH}^{+}\right) ;{ }^{1} \mathrm{H}-\mathrm{NMR}$ (DMSO-d $) \delta 6.36(\mathrm{~d}, 1 \mathrm{H}, \mathrm{C}-7 \mathrm{H}), 7.37(\mathrm{t}$, $1 \mathrm{H}, J=2.5 \mathrm{~Hz}, \mathrm{C}-6 \mathrm{H}), 7.78(\mathrm{~s}, 1 \mathrm{H}, \mathrm{C}-2 \mathrm{H}), 11.86(\mathrm{~s}, 1 \mathrm{H}), 12.08(\mathrm{~s}, 1 \mathrm{H})$.

7-Benzoyl-1H,3H,5H-pyrrolo[3,2-d]pyrimidin-2,4-dione (4): $75 \%$ yield from method A-1; mp $340{ }^{\circ} \mathrm{C}$ (decomposed); MS m/z, $255\left(\mathrm{M}^{+}\right) ;{ }^{1} \mathrm{H}-\mathrm{NMR}$ $\left(\mathrm{DMSO}-d_{6}\right) \delta 7.55\left(\mathrm{t}, 2 \mathrm{H}, J=7.5 \mathrm{~Hz}, \mathrm{C}^{\prime}-3 \mathrm{H}, \mathrm{C}^{\prime}-5 \mathrm{H}\right), 7.64(\mathrm{t}, 1 \mathrm{H}, J=6.5 \mathrm{~Hz}$, $\left.\mathrm{C}^{\prime}-4 \mathrm{H}\right), 7.68$ (d, 1H, J=4 Hz, C-6H), 7.82 (d, 2H, J=7 Hz, C'-2H, C'-6H), $10.0(\mathrm{~b}, 1 \mathrm{H}, \mathrm{OH}), 10.9(\mathrm{~b}, 1 \mathrm{H}, \mathrm{OH})$, Anal. Calcd for $\mathrm{C}_{13} \mathrm{H}_{9} \mathrm{~N}_{3} \mathrm{O}_{3} \cdot 1 / 2 \mathrm{H}_{2} \mathrm{O}: \mathrm{C}$, 59.03; H, 3.78; N, 15.90. Found: C, 59.00; H, 3.57; N, 15.92.

7-(p-Methylbenzoyl)-1H,3H,5H-pyrrolo[3,2-d]pyrimidin-2,4-dione $\quad(5)$ : $79 \%$ yield from method A-1; mp $>300{ }^{\circ} \mathrm{C}$; MS $m / z, 270\left(\mathrm{M}^{+}\right) ;{ }^{1} \mathrm{H}-\mathrm{NMR}$ $\left(\mathrm{DMSO}-d_{6}\right) \delta 2.37\left(\mathrm{~s}, 3 \mathrm{H}, \mathrm{CH}_{3}\right), 7.33\left(\mathrm{~d}, 2 \mathrm{H}, J=8 \mathrm{~Hz}, \mathrm{C}^{\prime}-3 \mathrm{H}, \mathrm{C}^{\prime}-5 \mathrm{H}\right), 7.68$ (s, 1H, C-6H), 7.73 (d, 2H, J=8 Hz, C'-2H, C'-6H). $10.3(\mathrm{~s}, 1 \mathrm{H}, \mathrm{OH}), 11.0$ (s, $1 \mathrm{H}, \mathrm{OH}), 12.85(\mathrm{~b}, 1 \mathrm{H}, \mathrm{NH})$. Anal. Calcd for $\mathrm{C}_{14} \mathrm{H}_{11} \mathrm{~N}_{3} \mathrm{O}_{3} \cdot 1 / 3 \mathrm{CF}_{3} \mathrm{SO}_{3} \mathrm{H}$ : C, 53.87; H, 3.76; N, 13.16. Found: C, 54.11; H, 3.93; N, 12.93 .

7-(3',4'-Dichlorobenzoyl)-1H,3H,5H-pyrrolo[3,2-d]pyrimidin-2,4-dione (6): $90 \%$ yield from method $\mathrm{A}-1$; mp $326^{\circ} \mathrm{C}$ (decomposed); MS $m / z, 324$ $\left(\mathrm{MH}^{+}\right) ;{ }^{1} \mathrm{H}-\mathrm{NMR}\left(\mathrm{DMSO}-d_{6}\right) \delta 7.77\left(\mathrm{~m}, 3 \mathrm{H}, \mathrm{C}-6 \mathrm{H}, \mathrm{C}^{\prime}-5 \mathrm{H}, \mathrm{C}^{\prime}-6 \mathrm{H}\right), 7.95(\mathrm{~s}$, $\left.1 \mathrm{H}, \mathrm{C}^{\prime}-4 \mathrm{H}\right), 10.19(\mathrm{~s}, 1 \mathrm{H}, \mathrm{OH}), 11.05(\mathrm{~s}, 1 \mathrm{H}, \mathrm{OH}), 12.9$ (s, 1H, NH); Anal. Calcd for $\mathrm{C}_{13} \mathrm{H}_{6} \mathrm{Cl}_{2} \mathrm{~N}_{3} \mathrm{O}_{3} \cdot 2 / 3 \mathrm{H}_{2} \mathrm{O}: \mathrm{C}, 46.41 ; \mathrm{H}, 2.47 ; \mathrm{N}, 12.50$. Found: $\mathrm{C}$, $46.20 ; \mathrm{H}, 2.53 ; \mathrm{N}, 12.24$
7-Benzyl-1H,3H,5H-pyrrolo[3,2-d]pyrimidin-2,4-dione (7): 11\% yield from method B, $77.6 \%$ yield from method $\mathrm{C} ; \mathrm{mp} 257^{\circ} \mathrm{C}$ (decomposed); MS $\mathrm{m} / \mathrm{z}$, $241\left(\mathrm{M}^{+}\right)$; ${ }^{1} \mathrm{H}-\mathrm{NMR}\left(\mathrm{DMSO}-d_{6}\right) \delta 3.79\left(\mathrm{~s}, 2 \mathrm{H}, \mathrm{CH}_{2}\right), 6.93(\mathrm{~d}, 1 \mathrm{H}, J=6 \mathrm{~Hz}$, C-6H), 7.15 (t, $\left.1 \mathrm{H}, J=6.5 \mathrm{~Hz}, \mathrm{C}^{\prime}-4 \mathrm{H}\right), 7.26\left(\mathrm{~m}, 4 \mathrm{H}, \mathrm{C}^{\prime}-2 \mathrm{H}, \mathrm{C}^{\prime}-3 \mathrm{H}, \mathrm{C}^{\prime}-5 \mathrm{H}\right.$, $\left.\mathrm{C}^{\prime}-6 \mathrm{H}\right), 10.58(\mathrm{~s}, 1 \mathrm{H}, \mathrm{OH}), 10.87$ (s,1H, OH), 11.65 (s, 1H, NH). Anal. Calcd for $\mathrm{C}_{13} \mathrm{H}_{11} \mathrm{~N}_{3} \mathrm{O}_{2} \cdot 1 / 15 \mathrm{CF}_{3} \mathrm{SO}_{3} \mathrm{H}$ : C, 62.41; H, 4.40; N, 16.72. Found: C, 62.37; H, 4.12; N, 16.37 .

7-(p-Methylbenzyl)-1H,3H,5H-pyrrolo[3,2-d]pyrimidin-2,4-dione (8): $58 \%$ yield from method $\mathrm{C}, 17 \%$ yield from method $\mathrm{B} ; \mathrm{mp} 283^{\circ} \mathrm{C}$ (decomposed); MS $m / z, 256\left(\mathrm{MH}^{+}\right) ;{ }^{1} \mathrm{H}-\mathrm{NMR}\left(\mathrm{DMSO}-d_{6}\right) \delta 2.22\left(\mathrm{~S}, 3 \mathrm{H}, \mathrm{CH}_{3}\right), 3.72(\mathrm{~s}, 2 \mathrm{H}$, $\left.\mathrm{CH}_{2}\right), 6.88(\mathrm{~s}, 1 \mathrm{H}, \mathrm{C}-6 \mathrm{H}), 7.0-7.2(\mathrm{~m}, 5 \mathrm{H}$, aromatic $\mathrm{H}), 10.57(\mathrm{~s}, 1 \mathrm{H}, \mathrm{OH})$, $10.82(\mathrm{~s}, 1 \mathrm{H}, \mathrm{OH}), 11.62(\mathrm{~s}, 1 \mathrm{H}, \mathrm{NH})$. Anal. Calcd for $\mathrm{C}_{14} \mathrm{H}_{13} \mathrm{~N}_{3} \mathrm{O}_{2} \cdot 1 / 2 \mathrm{H}_{2} \mathrm{O}$ : C, 63.57; H, 5.30; N, 15.90, Found: C, 63.95; H, 4.94; N, 15.64.

7-(3',4'-Dichlorobenzyl)- $1 H, 3 H, 5 H$-pyrrolo[3,2-d]pyrimidin-2,4-dione (9): $8 \%$ yield from method $\mathrm{B}$; mp $278^{\circ} \mathrm{C}$ (decomposed); MS $\mathrm{m} / \mathrm{z}, 310$ $\left(\mathrm{MH}^{+}\right) ;{ }^{1} \mathrm{H}-\mathrm{NMR}\left(\mathrm{DMSO}-d_{6}\right) \delta 3.79\left(\mathrm{~s}, 2 \mathrm{H}, \mathrm{CH}_{2}\right), 7.07(\mathrm{~d}, 1 \mathrm{H}, J=3 \mathrm{~Hz}, \mathrm{C}-$ $6 \mathrm{H}), 7.23\left(\mathrm{dd}, 1 \mathrm{H}, J=1.5 \mathrm{~Hz}, \mathrm{C}^{\prime}-6 \mathrm{H}\right), 7.51\left(\mathrm{~s}, 1 \mathrm{H}, \mathrm{C}^{\prime}-2 \mathrm{H}\right), 7.53(\mathrm{~d}, 1 \mathrm{H}$, $\left.J=1.5 \mathrm{~Hz}, \mathrm{C}^{\prime}-5 \mathrm{H}\right), 10.60$ (s, 1H, OH), 10.88 (s, 1H, OH), 11.76 (s, 1H, NH). Anal. Calcd for $\mathrm{C}_{13} \mathrm{H}_{9} \mathrm{Cl}_{2} \mathrm{~N}_{3} \mathrm{O}_{2} \cdot 1 / 9 \mathrm{H}_{2} \mathrm{O}: \mathrm{C}, 50.02 ; \mathrm{H}, 2.98 ; \mathrm{N}, 13.46$. Found: C, 50.33; H, 2.96; N, 13.07 .

2-Amino-3H,5H-7-benzoylpyrrolo[3,2-d]pyrimidin-4-one (10): $54 \%$ yield from A-2; mp $287^{\circ} \mathrm{C}$ (decomposed, lit. ${ }^{18)} \mathrm{mp}>250{ }^{\circ} \mathrm{C}$ ); $\mathrm{MS} m / z, 255$ $\left(\mathrm{MH}^{+}\right) ;{ }^{1} \mathrm{H}-\mathrm{NMR}\left(\mathrm{DMSO}-d_{6}\right) \delta 6.4\left(\mathrm{~b}, 2 \mathrm{H}, \mathrm{NH}_{2}\right), 7.49\left(\mathrm{t}, 2 \mathrm{H}, J=7.5 \mathrm{~Hz}, \mathrm{C}^{\prime}-\right.$ $\left.3 \mathrm{H}, \mathrm{C}^{\prime}-5 \mathrm{H}\right), 7.56(\mathrm{~s}, 1 \mathrm{H}, \mathrm{C}-6 \mathrm{H}), 7.6\left(\mathrm{t}, 1 \mathrm{H}, \mathrm{C}^{\prime}-4 \mathrm{H}\right), 7.79(\mathrm{~d}, 2 \mathrm{H}, J=7.0 \mathrm{~Hz}$, $\left.\mathrm{C}^{\prime}-2 \mathrm{H}, \mathrm{C}^{\prime}-6 \mathrm{H}\right)$. Anal. Calcd for $\mathrm{C}_{13} \mathrm{H}_{10} \mathrm{~N}_{4} \mathrm{O}_{2} \cdot 1 / 6 \mathrm{CF}_{3} \mathrm{SO}_{3} \mathrm{H}: \mathrm{C}, 56.18 ; \mathrm{H}$, 3.94; N, 19.91. Found: C, 56.58; H, 3.64; N, 20.06 .

2-Amino-3H,5H-7-( $p$-methylbenzoyl)pyrrolo[3,2- $d$ ]pyrimidin-4-one (11): $55 \%$ yield from method $\mathrm{A}-2$; mp $267^{\circ} \mathrm{C}$ (decomposed); MS $\mathrm{m} / \mathrm{z}, 269$ $\left(\mathrm{MH}^{+}\right) ;{ }^{1} \mathrm{H}-\mathrm{NMR}\left(\mathrm{DMSO}-d_{6}\right) \delta 6.4\left(\mathrm{~b}, 2 \mathrm{H}, \mathrm{NH}_{2}\right), 7.30\left(\mathrm{~d}, 2 \mathrm{H}, J=8 \mathrm{~Hz}, \mathrm{C}^{\prime}-\right.$ $\left.3 \mathrm{H}, \mathrm{C}^{\prime}-5 \mathrm{H}\right), 7.55$ (s, 1H, C-6H), $7.71\left(\mathrm{~d}, 2 \mathrm{H}, J=8 \mathrm{~Hz}, \mathrm{C}^{\prime}-2 \mathrm{H}, \mathrm{C}^{\prime}-6 \mathrm{H}\right), 10.9$ (b, $1 \mathrm{H}, \mathrm{OH}), 12.46(\mathrm{~b}, 1 \mathrm{H}, \mathrm{NH})$. Anal. Calcd for $\mathrm{C}_{14} \mathrm{H}_{12} \mathrm{~N}_{4} \mathrm{O}_{2} \cdot 1 / 5 \mathrm{CF}_{3} \mathrm{SO}_{3} \mathrm{H}$ : C, 57.12; H, 4.09; N, 18.78. Found: C, 57.21; H, 4.44; N, 18.44.

2-Amino-3H,5H-7-(3',4'-dichlorobenzoyl)pyrrolo[3,2-d]pyrimidin-4-one (12): $91 \%$ yield from method $\mathrm{A}-2 ; \mathrm{mp} 310^{\circ} \mathrm{C}$ (decomposed); MS $\mathrm{m} / \mathrm{z}, 323$ $\left(\mathrm{M}^{+}\right) ;{ }^{1} \mathrm{H}-\mathrm{NMR}\left(\mathrm{DMSO}-d_{6}\right) \delta 6.8\left(\mathrm{~s}, 2 \mathrm{H}, \mathrm{NH}_{2}\right), 7.6(\mathrm{~s}, 1 \mathrm{H}, \mathrm{C}-6 \mathrm{H}), 7.78(\mathrm{~d}$, $\left.2 \mathrm{H}, \mathrm{C}^{\prime}-5 \mathrm{H}, \mathrm{C}^{\prime}-6 \mathrm{H}\right), 7.96\left(\mathrm{~s}, 1 \mathrm{H}, \mathrm{C}^{\prime}-2 \mathrm{H}\right), 10.18(\mathrm{~s}, 1 \mathrm{H}, \mathrm{OH}), 11.03(\mathrm{~s}, 1 \mathrm{H}$, $\mathrm{NH})$; Anal. Calcd for $\mathrm{C}_{13} \mathrm{H}_{8} \mathrm{Cl}_{2} \mathrm{~N}_{4} \mathrm{O}_{2} \cdot 1.6 \mathrm{H}_{2} \mathrm{O}: \mathrm{C}, 44.36 ; \mathrm{H}, 3.21 ; \mathrm{N}, 15.92$. Found: C, 44.27; H, 2.82; N, 15.66 .

2-Amino-3H,5H-7-(3',5'-dichlorobenzoyl)pyrrolo[3,2- $d]$ pyrimidin-4-one (13): $57 \%$ yield from method A-2; mp $308^{\circ} \mathrm{C}$ (decomposed); MS $m / z, 324$ $\left(\mathrm{MH}^{+}\right) ;{ }^{1} \mathrm{H}-\mathrm{NMR}\left(\mathrm{DMSO}-d_{6}\right) \delta 6.41\left(\mathrm{~b}, 2 \mathrm{H}, \mathrm{NH}_{2}\right), 7.70(\mathrm{~s}, 1 \mathrm{H}, \mathrm{C}-6 \mathrm{H}), 7.75$ (s, 2H, C'-2H, C'-6H), $7.94\left(\mathrm{~s}, 1 \mathrm{H}, \mathrm{C}^{\prime}-4 \mathrm{H}\right), 11.03(\mathrm{~b}, 1 \mathrm{H}, \mathrm{OH}), 12.6(\mathrm{~s}, 1 \mathrm{H}$, $\mathrm{NH})$. Anal. Calcd for $\mathrm{C}_{13} \mathrm{H}_{8} \mathrm{Cl}_{2} \mathrm{~N}_{4} \mathrm{O}_{2} \cdot 2 / 3 \mathrm{CH}_{3} \mathrm{OH} \cdot 1 / 15 \mathrm{CF}_{3} \mathrm{SO}_{3} \mathrm{H}$ : C, 46.49; $\mathrm{H}, 3.02 ; \mathrm{N}, 15.79$. Found: C, 46.22; H, 2.91; N, 15.61.

2-Amino-3H,5H-7-(m-nitrobenzoyl)pyrrolo[3,2- $d$ pyrimidin-4-one (14): $72 \%$ yield from method A-2; mp $>300{ }^{\circ} \mathrm{C}$; MS $m / z, 300\left(\mathrm{MH}^{+}\right) ;{ }^{1} \mathrm{H}-\mathrm{NMR}$ $\left(\mathrm{DMSO}-d_{6}\right) \delta 6.36\left(\mathrm{~b}, 2 \mathrm{H}, \mathrm{NH}_{2}\right), 7.57(\mathrm{~s}, 1 \mathrm{H}, \mathrm{C}-6 \mathrm{H}), 7.77(\mathrm{t}, 1 \mathrm{H}, J=7.5 \mathrm{~Hz}$, $\left.\mathrm{C}^{\prime}-5 \mathrm{H}\right), 8.19,\left(\mathrm{~d}, 1 \mathrm{H}, J=7.5 \mathrm{~Hz}, \mathrm{C}^{\prime}-6 \mathrm{H}\right), 8.39\left(\mathrm{~d}, 1 \mathrm{H}, J=8.0 \mathrm{~Hz}, \mathrm{C}^{\prime}-4 \mathrm{H}\right)$, $8.51\left(\mathrm{~s}, 1 \mathrm{H}, \mathrm{C}^{\prime}-2 \mathrm{H}\right)$. Anal. Calcd for $\mathrm{C}_{13} \mathrm{H}_{9} \mathrm{~N}_{5} \mathrm{O}_{4} \cdot 0.4 \mathrm{CH}_{3} \mathrm{OH} \cdot 0.15 \mathrm{CF}_{3} \mathrm{SO}_{3} \mathrm{H}$ : C, 48.60; H, 3.21; N, 20.93. Found: C, 48.57; H, 2.91; N, 20.67.

2-Amino-3H,5H-7-( $p$-hydroxylbenzoyl)pyrrolo[3,2- $d]$ pyrimidin-4-one (15): $75 \%$ yield from method $\mathrm{A}-2$ at $100^{\circ} \mathrm{C}$ for $3 \mathrm{~d}$; mp $>300^{\circ} \mathrm{C}$; MS $m / z$, $271\left(\mathrm{MH}^{+}\right)$; ${ }^{1} \mathrm{H}-\mathrm{NMR}\left(\mathrm{DMSO}-d_{6}\right) \delta 6.85\left(\mathrm{~d}, 2 \mathrm{H}, d=8.5 \mathrm{~Hz}, \mathrm{C}^{\prime}-3 \mathrm{H}, \mathrm{C}^{\prime}-5 \mathrm{H}\right)$, $7.62(\mathrm{~s}, 1 \mathrm{H}, \mathrm{C}-6 \mathrm{H}), 7.75$ (d, 2H, J=8 Hz, C'-2H, C'-6H), 10.27 (s, 1H, OH), 12.54 (b, $1 \mathrm{H}, \mathrm{NH})$. Anal. Calcd for $\mathrm{C}_{13} \mathrm{H}_{10} \mathrm{~N}_{4} \mathrm{O}_{3} \cdot \mathrm{CH}_{3} \mathrm{COCH}_{3} \cdot 0.65 \mathrm{CF}_{3} \mathrm{SO}_{3} \mathrm{H}$ : C, 46.95; H, 3.94; N, 13.16. Found: C, 47.32; H, 3.81; N, 12.94.

2-Amino-3H,5H-7-benzylpyrrolo[3,2- $d$ ]pyrimidin-4-one (16): $20 \%$ yield from method $\mathrm{B}, 53 \%$ yield from method $\mathrm{C}$; mp $255^{\circ} \mathrm{C}$ (decomposed, lit. ${ }^{3)}$ $269-270{ }^{\circ} \mathrm{C}$ ); $\mathrm{MS} m / z, 240\left(\mathrm{M}^{+}\right) ;{ }^{1} \mathrm{H}-\mathrm{NMR}\left(\mathrm{DMSO}-d_{6}\right) \delta 3.77(\mathrm{~s}, 2 \mathrm{H}$, $\left.\mathrm{CH}_{2}\right), 5.79$ (b, $\left.2 \mathrm{H}, \mathrm{NH}_{2}\right), 6.90(\mathrm{~s}, 1 \mathrm{H}, \mathrm{C}-6 \mathrm{H}), 7.0-7.3(\mathrm{~m}, 5 \mathrm{H}$, aromatic $\mathrm{H})$, 10.25 (b, 1H, OH), $11.2(\mathrm{~b}, 1 \mathrm{H}, \mathrm{NH})$.

2-Amino-3H,5H-7-(p-methylbenzyl)pyrrolo[3,2- $d$ ]pyrimidin-4-one (17): $12 \%$ yield from method $\mathrm{B}$; mp $175^{\circ} \mathrm{C}$ (decomposed); MS m/z, $254\left(\mathrm{M}^{+}\right)$; ${ }^{1} \mathrm{H}-\mathrm{NMR}\left(\mathrm{DMSO}-d_{6}\right) \delta 2.22\left(\mathrm{~s}, 3 \mathrm{H}, \mathrm{CH}_{3}\right), 3.75\left(\mathrm{~s}, 2 \mathrm{H}, \mathrm{CH}_{2}\right), 5.8(\mathrm{~b}, 2 \mathrm{H}$, $\left.\mathrm{NH}_{2}\right), 6.90(\mathrm{~d}, 1 \mathrm{H}, J=3 \mathrm{~Hz}, \mathrm{C}-6 \mathrm{H}), 7.04\left(\mathrm{~d}, 2 \mathrm{H}, J=7.5 \mathrm{~Hz}, \mathrm{C}^{\prime}-3 \mathrm{H}, \mathrm{C}^{\prime}-5 \mathrm{H}\right)$, $7.10\left(\mathrm{~d}, 2 \mathrm{H}, J=8 \mathrm{~Hz}, \mathrm{C}^{\prime}-2 \mathrm{H}, \mathrm{C}^{\prime}-6 \mathrm{H}\right), 10.32(\mathrm{~s}, 1 \mathrm{H}, \mathrm{NH}), 11.2(1 \mathrm{H}, \mathrm{NH})$. Anal. Calcd for $\mathrm{C}_{14} \mathrm{H}_{14} \mathrm{~N}_{4} \mathrm{O} \cdot 2 / 3 \mathrm{CH}_{3} \mathrm{COOH}$ : C, 62.52; H, 5.66; N, 19.03 . Found: C, 62.40; H, 5.62; N, 19.20.

2-Amino-3H,5H-7-(3', $4^{\prime}$-dichlorobenzyl)pyrrolo[3,2-d]pyrimidin-4-one (18): $14 \%$ yield from method $\mathrm{B}$; mp $245^{\circ} \mathrm{C}$ (decomposed, lit. ${ }^{3)} 278$ $280{ }^{\circ} \mathrm{C}$ ); MS $m / z, 309\left(\mathrm{MH}^{+}\right)$; ${ }^{1} \mathrm{H}-\mathrm{NMR}$ (DMSO-d $) \delta 3.79\left(\mathrm{~s}, 2 \mathrm{H}, \mathrm{CH}_{2}\right.$ ), $5.82\left(\mathrm{~b}, 2 \mathrm{H}, \mathrm{NH}_{2}\right), 6.99(\mathrm{~d}, 1 \mathrm{H}, \mathrm{C}-6 \mathrm{H}), 7.21\left(\mathrm{~m}, 1 \mathrm{H}, \mathrm{C}^{\prime}-6 \mathrm{H}\right), 7.43(\mathrm{~m}, 1 \mathrm{H}$, $\left.\mathrm{C}^{\prime}-2 \mathrm{H}\right), 7.46\left(\mathrm{~m}, 1 \mathrm{H}, \mathrm{C}^{\prime}-5 \mathrm{H}\right), 10.38(\mathrm{~s}, 1 \mathrm{H}, \mathrm{OH}), 11.28(\mathrm{~s}, 1 \mathrm{H}, \mathrm{OH})$.

2-Amino-3H,5H-7-(m-nitrobenzyl)pyrrolo[3,2- $d$ ]pyrimidin-4-one (19): 15\% 
yield from method $\mathrm{B}$ in the solvent of triflic acid; $\mathrm{mp} 190^{\circ} \mathrm{C}$; $\mathrm{MS} \mathrm{m} / \mathrm{z}, 285$ $\left(\mathrm{M}^{+}\right) ;{ }^{1} \mathrm{H}-\mathrm{NMR}\left(\mathrm{DMSO}-d_{6}\right) \delta 4.05\left(\mathrm{~s}, 2 \mathrm{H}, \mathrm{CH}_{2}\right), 7.33(\mathrm{~s}, 2 \mathrm{H}, \mathrm{C}-6 \mathrm{H}), 7.57$ (t, $\left.1 \mathrm{H}, \mathrm{C}^{\prime}-5 \mathrm{H}\right), 7.70\left(\mathrm{~d}, 1 \mathrm{H}, J=6.9 \mathrm{~Hz}, \mathrm{C}^{\prime}-6 \mathrm{H}\right), 8.06\left(\mathrm{~d}, 1 \mathrm{H}, J=7.5 \mathrm{~Hz}, \mathrm{C}^{\prime}-4 \mathrm{H}\right)$, $8.11\left(\mathrm{~s}, 1 \mathrm{H}, \mathrm{C}^{\prime}-2 \mathrm{H}\right), 12.4(\mathrm{~s}, 1 \mathrm{H}, \mathrm{NH})$. Anal. Calcd for $\mathrm{C}_{13} \mathrm{H}_{11} \mathrm{~N}_{5} \mathrm{O}_{3}$. $1.4 \mathrm{CF}_{3} \mathrm{COOH} \cdot 0.5 \mathrm{H}_{2} \mathrm{O}: \mathrm{C}, 41.81 ; \mathrm{H}, 3.28 ; \mathrm{N}, 15.43$. Found: $\mathrm{C}, 41.80 ; \mathrm{H}$, $3.15 ; \mathrm{N}, 15.27$.

2-Amino-3H,5H-7-( $p$-methoxybenzyl)pyrrolo[3,2-d]pyrimidin-4-one (20): $21 \%$ yield from method $\mathrm{B}$ using $p$-methoxybenzyl bromide as alkylating agents at $80^{\circ} \mathrm{C}$ for $24 \mathrm{~h} ; \mathrm{mp} 275^{\circ} \mathrm{C}$, decomposed; ${ }^{1} \mathrm{H}-\mathrm{NMR}$ (DMSO- $d_{6}$ ) $\delta 3.68\left(\mathrm{~s}, 3 \mathrm{H}, \mathrm{OCH}_{3}\right), 3.71\left(\mathrm{~s}, 2 \mathrm{H}, \mathrm{CH}_{2}\right), 5.82\left(\mathrm{~s}, 2 \mathrm{H}, \mathrm{NH}_{2}\right), 6.78(\mathrm{~d}, 2 \mathrm{H}$, $\left.J=8.5 \mathrm{~Hz}, \mathrm{C}^{\prime}-3, \mathrm{C}^{\prime}-5 \mathrm{H}\right), 6.86(\mathrm{~d}, 1 \mathrm{H}, J=2.5 \mathrm{~Hz}, \mathrm{C}-6 \mathrm{H}), 7.13(\mathrm{~d}, 2 \mathrm{H}$, $\left.J=9 \mathrm{~Hz}, \mathrm{C}^{\prime}-2 \mathrm{H}, \mathrm{C}^{\prime}-6 \mathrm{H}\right), 10.4$ (b, 1H, NH), 11.19 (s, 1H, NH). Anal. Calcd for $\mathrm{C}_{14} \mathrm{H}_{14} \mathrm{~N}_{4} \mathrm{O}_{2} \cdot 0.8 \mathrm{CH}_{3} \mathrm{COOH}$ : C, 58.85; H, 5.46; N, 17.60. Found: $\mathrm{C}$, $59.22 ; \mathrm{H}, 5.17 ; \mathrm{N}, 17.27$.

7-Benzoyl-3H,5H-pyrrolo[3,2- $d$ ]pyrimidin-4-one (21): $13 \%$ yield from method A-1; mp $>300{ }^{\circ} \mathrm{C}$; MS $m / z, 239\left(\mathrm{M}^{+}\right) ;{ }^{1} \mathrm{H}-\mathrm{NMR}$ (DMSO- $d_{6}$ ) $\delta 7.49$ (t, $\left.2 \mathrm{H}, J=7 \mathrm{~Hz}, \mathrm{C}^{\prime}-3 \mathrm{H}, \mathrm{C}^{\prime}-5 \mathrm{H}\right), 7.59$ (t, $\left.1 \mathrm{H}, J=7.5 \mathrm{~Hz}, \mathrm{C}^{\prime}-4 \mathrm{H}\right), 7.79(\mathrm{~d}, 2 \mathrm{H}$, $\left.J=7.5 \mathrm{~Hz}, \mathrm{C}^{\prime}-2 \mathrm{H}, \mathrm{C}^{\prime}-6 \mathrm{H}\right), 7.86(\mathrm{~d}, 1 \mathrm{H}, J=3.5 \mathrm{~Hz}, \mathrm{C}-6 \mathrm{H}), 7.91$ (s, $1 \mathrm{H}, \mathrm{C}-$ $2 \mathrm{H})$. Anal. Calcd for $\mathrm{C}_{13} \mathrm{H}_{9} \mathrm{~N}_{3} \mathrm{O}_{2} \cdot 0.1 \mathrm{CF}_{3} \mathrm{SO}_{3} \mathrm{H}: \mathrm{C}, 61.88 ; \mathrm{H}, 3.61 ; \mathrm{N}, 16.53$. Found: C, $62.18 ; \mathrm{H}, 3.58 ; \mathrm{N}, 16.31$.

7-(3',4'-Dichlorobenzoyl)-3H,5H-pyrrolo[3,2- $d]$ pyrimidin-4-one $\quad$ (22): $15 \%$ yield form method A-1; mp $290{ }^{\circ} \mathrm{C}$ (decomposed); MS m/z 308 $\left(\mathrm{MH}^{+}\right)$; ${ }^{1} \mathrm{H}-\mathrm{NMR}$ (DMSO- $\left.\left.d_{6}\right) \delta 7.75\left(\mathrm{~m}, 2 \mathrm{H}, \mathrm{C}^{\prime}-5 \mathrm{H}, \mathrm{C}^{\prime}-6 \mathrm{H}\right)\right), 7.90(\mathrm{~s}, 1 \mathrm{H}$, $\mathrm{C}-6 \mathrm{H}), 7.95(\mathrm{~s}, 1 \mathrm{H}, \mathrm{C}-2 \mathrm{H}), 7.98\left(\mathrm{~s}, 1 \mathrm{H}, \mathrm{C}^{\prime}-2 \mathrm{H}\right)$. Anal. Calcd for $\mathrm{C}_{13} \mathrm{H}_{7} \mathrm{~N}_{3} \mathrm{O}_{2} \mathrm{Cl}_{2} \cdot 0.12 \mathrm{CF}_{3} \mathrm{SO}_{3} \mathrm{H}: \mathrm{C}, 48.31 ; \mathrm{H}, 2.20 ; \mathrm{N}, 12.88$. Found: $\mathrm{C}, 48.29$; $\mathrm{H}, 2.24 ; \mathrm{N}, 12.51$.

7-Benzyl-9-3H,5H-pyrrolo[3,2-d]pyrimidin-4-one (23): $20 \%$ yield from method B; mp $249^{\circ} \mathrm{C}$ (decomposed, lit. ${ }^{6} 340^{\circ} \mathrm{C}$, decomposed); MS $\mathrm{m} / \mathrm{z}$, $226\left(\mathrm{MH}^{+}\right) ;{ }^{1} \mathrm{H}-\mathrm{NMR}\left(\mathrm{DMSO}-d_{6}\right) \delta 3.91\left(\mathrm{~s}, 2 \mathrm{H}, \mathrm{CH}_{2}\right), 7.3(\mathrm{~m}, 6 \mathrm{H}, \mathrm{C}-6 \mathrm{H}$, aromatic $\mathrm{H}), 7.78(\mathrm{~s}, 1 \mathrm{H}, \mathrm{C}-2 \mathrm{H})$.

7-(3',4'-Dichlorobenzyl)-3H,5H-pyrrolo[3,2- $d]$ pyrimidin-4-one (24): 19\% yield from method B; mp $275^{\circ} \mathrm{C}$ (decomposed); MS m/z, $294\left(\mathrm{MH}^{+}\right) ;{ }^{1} \mathrm{H}-$ NMR (DMSO- $\left.d_{6}\right) \delta 3.93\left(\mathrm{~s}, 2 \mathrm{H}, \mathrm{CH}_{2}\right), 7.26\left(\mathrm{~m}, 2 \mathrm{H}, \mathrm{C}-6 \mathrm{H}, \mathrm{C}^{\prime}-6 \mathrm{H}\right), 7.50(\mathrm{~m}$, $\left.2 \mathrm{H}, \mathrm{C}^{\prime}-2 \mathrm{H}, \mathrm{C}^{\prime}-5 \mathrm{H}\right), 7.78$ (s, $\left.1 \mathrm{H}, \mathrm{C}-2 \mathrm{H}\right)$. Anal. Calcd for $\mathrm{C}_{13} \mathrm{H}_{9} \mathrm{Cl}_{2} \mathrm{~N}_{3} \mathrm{O}$. $1 / 2 \mathrm{H}_{2} \mathrm{O}: \mathrm{C}, 53.30 ; \mathrm{H}, 3.17$; N, 13.34. Found: C, 52.94; H, 3.09; N, 13.69 .

\section{References}

1) Carson D. A., Carrera C. J., Semin Hematol., 27, 260-269 (1990).

2) Gilbertsen R. B., Josyula U., Sircar J. C., Dong M. K., Wu W. S., Wilburn D. J., Conroy M. C., Biochem. Pharmacol., 44, 996-999 (1992).

3) Montgomery J. A., Niwas S., Rose J. D., Secrist 3rd J. A., Babu Y. S., Bugg C. E., Erion M. D., Guida W. C., Ealick S. E., J. Med. Chem., 36, 55-69 (1993).

4) Secrist 3rd J. A., Niwas S., Rose J. D., Babu Y. S., Bugg C. E., Erion M. D., Guida W. C., Ealick S. E., Montgomery J. A., J. Med. Chem., 36, 1847-1854 (1993).

5) Erion M. D., Niwas S., Rose J. D., Ananthan S., Allen M., Secrist 3rd J. A., Babu Y. S., Bugg C. E., Guida W. C., Ealick S. E., Montgomery J. A., J. Med. Chem., 36, 3771-3783 (1993).

6) Niwas S., Chand P., Pathak V. P., Montgomery J. A., J. Med. Chem., 37, 2447-2480 (1994).

7) Sircar J. C., Kostlan C. R., Gilbertsen R. B., Bennett M. K., Dong M. K., Cetenko W. J., J. Med. Chem., 36, 1605-1609 (1992).

8) Klein R. S., Lim M.-I., Tam S. Y.-K., Fox J. J., J. Org. Chem., 43, 2536-2539 (1978).

9) Imai K. I., Chem. Pharm. Bull., 12, 1030-1042 (1964).

10) Girgis N. S., Michael M. A., Smee D. F., Alaghamandan H. A., Robins R. K., Cottam H. B., J. Med. Chem., 33, 2750-2755 (1990).

11) a) Rand L., Dolinski R., J. Org. Chem., 31, 3063-3065 (1966); b) Idem, ibid., 31, 4061-4066 (1966).

12) Brown F. J., Cronk L. A., Aharony D., Snyder D. W., J. Med. Chem., 35, 2419-2439 (1992).

13) Shen Y.-S., Liu H.-X., Wu M., Du W.-Q., Chen Y.-Q., Li N.-P., J. Org. Chem., 56, 7160-7162 (1991).

14) Kife W. K., Ranganathan P., Zeldin M., J. Org. Chem., 55, 56105613 (1990).

15) Furneaux R. H., Tyler P. C., J. Org. Chem., 64, 8411-8412 (1999).

16) Brakta M., Daves G. D., Jr., J. Chem. Soc., Perkin Trans. 1, 1992, 1883-1884.

17) Polonovski M., Pesson M., Bull. Soc. Chim. (France), 1948, 688-694.

18) Morris P. E., Jr., Elliott A. J., Walton S. P., Williams C. H., Montgomery J. A., Nucleoside, Nucleotides, Nucleic Acid, 19, 379-404 (2000). 